7th International Workshop on Astronomy and

Relativistic Astrophysics (IWARA 2016)

International Journal of Modern Physics: Conference Series

Vol. 45 (2017) 1760052 (7 pages)

(C) The Author(s)

DOI: $10.1142 / \mathrm{S} 2010194517600527$

\title{
The Gravitomagnetism in the Solar System
}

\author{
Flavia Rocha, Manuel Malheiro and Rubens Marinho Jr. \\ Departamento de Física, Instituto Tecnológico de Aeronáutica (ITA) \\ Vila das Acacias, 12228-900 São José dos Campos, SP, Brazil \\ rocha.pereira.flavia@gmail.com,malheiro@ita.br,marinho@ita.br
}

Published 15 August 2017

\begin{abstract}
In 1918, Joseph Lense and Hans Thirring discovered the gravitomagnetic (GM) effect of Einstein field equations in weak field and slow motion approximation. They showed that Einstein equations in this approximation can be written as in the same form as Maxwell's equation for electromagnetism. In these equations the charge and electric current are replaced by the mass density and the mass current. Thus, the gravitomagnetism formalism in astrophysical system is used with the mass assuming the role of the charge. In this work, we present the deduction of gravitoelectromagnetic equations and the analogue of the Lorentz force in the gravitomagnetism. We also discuss the problem of Mercury's perihelion advance orbit, we propose solutions using GM formalism using a dipole-dipole potential for the Sun-Planet interaction.
\end{abstract}

Keywords: General Relativity, Gravitomagnetism, Advance of Mercury's Perihelion.

PACS numbers: 04.80

\section{Introduction}

In 1916, Einstein published the General Relativity Theory (GR), describing how space-time is affected by the mass distribution present in the Universe. GR is a geometric theory of gravitation that extends Special Relativity to accelerated frames, introducing the principle that gravitational and inertial forces are equivalent.

The dynamics of General Relativity is described by a set of equations known as the Einstein field equations. These equations relate the geometric properties of space-time, described by a metric and its derivatives of first and second order with the energy-momentum tensor. The energy-moment tensor describes the matter distribution.

There are several exact solutions that have played a very important role in both theoretical and experimental development of GR ${ }^{1,2}$. One of the most important

This is an Open Access article published by World Scientific Publishing Company. It is distributed under the terms of the Creative Commons Attribution 4.0 (CC-BY) License. Further distribution of this work is permitted, provided the original work is properly cited. 
solutions of Einstein's field equations is the one obtained by Karl Schwarzchild in 1916 which represents the exterior of a matter-energy distribution with spherical symmetry.

Another important development was made by Lense and Thirring in 1918, when they solved Einstein's equations in the weak field and slow motion approximation for systems in rotation ${ }^{3,4}$. They found that linearizing the equations they can be written in four equations analogous to Maxwell's equations, but in the place of charge we have mass. A new concept arises, a mass current generates a gravitomagnetic field analogous to the magnetic field. These equations are called gravitoelectromagnetic equations.

In this work, we will show mathematically from Einstein's field equations, how we obtain the Gravitomagnetic equations and the analogous form of the Lorentz force. The gravitomagnetic approximation will be apply to the Solar System planet's.

\section{Gravitomagnetic Equations}

Einstein's field equations are non-linear and have a rather complex algebra. In weak gravitational fields, distant from astrophysical sources, space-time is approximately plane ${ }^{2}$. The calculation of the Einstein field equation far from the sources is done by a weak field approximation, which consists of considering that the metric is basically the Minkowski metric plus a disturbing term.

$$
g_{\mu \nu}=\eta_{\mu \nu}+h_{\mu \nu}
$$

where, $h_{\mu \nu}$ is the perturbation term in the space-time $\left(\left|h_{\mu \nu}\right| \ll 1,\left|\partial_{\rho} h_{\mu \nu}\right| \ll\right.$ $\left.1,\left|\partial_{\sigma} \partial_{\rho} h_{\mu \nu}\right| \ll 1, \ldots\right)$ and the Minkowski metric have the signature: $-1,1,1,1$.

Taking the weak field limit means that we will neglect the order terms $\left|h_{\mu \nu}\right|^{2}$ and greater in the following equation ${ }^{1}$. This implies that:

$$
g^{\mu \nu}=\eta^{\mu \nu}-h^{\mu \nu} .
$$

Now with the new metric set, we find the Christoffel symbols, the Ricci tensor, the scalar of curvature and the Einstein tensor, which is:

$$
G_{\mu \nu}=\frac{1}{2}\left(\partial_{\rho} \partial_{\nu} h_{\mu}^{\rho}-\partial_{\rho} \partial^{\rho} h_{\mu \nu}-\partial_{\mu} \partial_{\nu} h+\partial_{\mu} \partial_{\rho} h_{\nu}^{\rho}\right)-\frac{1}{2} \eta_{\mu \nu}\left(\partial_{\rho} \partial_{\sigma} h^{\sigma \rho}-\partial_{\rho} \partial^{\rho} h\right) .
$$

Now we will apply a coordinate transformation. We start by introducing the reverse trace tensor:

$$
\begin{aligned}
\bar{h}_{\mu \nu} & =h_{\mu \nu}-\frac{1}{2} \eta_{\mu \nu} h \quad(\bar{h}=-h), \\
h_{\mu \nu} & =\bar{h}_{\mu \nu}-\frac{1}{2} \eta_{\mu \nu} \bar{h} . .
\end{aligned}
$$

The field equations become

$$
\square \bar{h}_{\mu \nu}+\eta_{\mu \nu} \partial_{\rho} \partial_{\sigma} \bar{h}^{\sigma \rho}-\partial_{\nu} \partial_{\rho} \bar{h}_{\mu}^{\rho}-\partial_{\mu} \partial_{\rho} \bar{h}_{\nu}^{\rho}=-2 \kappa T_{\mu \nu}
$$


where, $\kappa=\frac{8 \pi G}{c^{4}}$ in CGS units. Fixing the Gauge condition

$$
\partial_{\sigma} \bar{h}^{\sigma \rho}=0
$$

follows,

$$
\square \bar{h}_{\mu \nu}=-2 \kappa T_{\mu \nu}
$$

\subsection{Slow Motion Approximation}

Now, we are going to make an approximation in which the source consists of a perfect non-relativistic fluid ${ }^{1}$. In this case, the pressure $p_{0}$ is negligible compared with the energy density $\rho_{0}$ and the four-velocity of the fluid $u^{\alpha}$ at each point will be such that $u^{0} \approx 1$ and $u^{i} \approx v^{i}<<1$, where Latin indices refer to the spatial part. In this approach, we maintain only the first order terms in $u^{i} \approx v^{i}$. For this case in which we have a low velocity approximation the components of the energy-moment tensor will be:

$$
\begin{aligned}
& T^{00} \approx \rho_{0} u^{0} u^{0} \approx \rho_{0}, \\
& T^{0 i}=T^{i 0} \approx \rho_{0} u^{0} u^{i} \approx \rho_{0} v^{i}, \\
& T^{i j} \approx \rho_{0} u^{i} u^{j} \approx 0 .
\end{aligned}
$$

In this approximation (8) can be written in four equation as

$$
\square \bar{h}_{0 \nu}=-2 \kappa T_{0 \nu}
$$

The gauge fixing condition reads:

$$
\partial_{\sigma} \bar{h}^{\sigma \rho}=0 \Rightarrow\left\{\begin{array}{l}
\partial_{\sigma} \bar{h}^{\sigma 0}=\partial_{0} \bar{h}^{00}+\partial_{i} \bar{h}^{i 0}=0 \\
\partial_{0} \bar{h}^{0 i}=0
\end{array}\right.
$$

The four field equations (12) can be rewritten as

$$
\left\{\begin{array} { l } 
{ \square \overline { h } _ { 0 0 } = - 2 \kappa T _ { 0 0 } } \\
{ \square \overline { h } _ { 0 i } = - 2 \kappa T _ { 0 i } }
\end{array} \Rightarrow \left\{\begin{array}{l}
\partial_{0}\left(\partial^{0} \bar{h}_{00}\right)+\partial_{i}\left(\partial^{i} \bar{h}_{00}\right)=-2 \kappa T_{00} \\
\partial_{0}\left(\partial^{0} \bar{h}_{0 i}\right)+\partial_{j}\left(\partial^{j} \bar{h}_{0 i}\right)=-2 \kappa T_{0 i}
\end{array}\right.\right.
$$

\subsection{Gravitoelectromagnetic fields}

We define the gravitoeletric potential $(\Phi)$, gravitomagnetic vector potential $\left(A^{i}\right)$, gravitational charge density $(\rho)$ and current $\left(j_{i}\right)$ according to ${ }^{1}$

$$
\Phi \equiv-\frac{c^{2} \bar{h}_{00}}{4}, \quad A^{i} \equiv-\frac{c^{2} \bar{h}_{0}^{i}}{4}, \quad \rho \equiv \frac{T_{00}}{c^{2}}, \quad j_{i} \equiv \frac{T_{0 i}}{c},
$$


Using the following definitions for gravitoelectric $\left(E^{i}\right)$ and gravitomagnetic $\left(B^{i}\right)$ fields:

$$
\begin{aligned}
E^{i} & \equiv-\partial^{i} \Phi+\frac{1}{2} \partial_{0} A^{i}=\frac{1}{4} \partial^{i} \bar{h}_{00}-\frac{1}{4} \partial_{0} \bar{h}_{0}^{i} \\
B^{n} & \equiv \varepsilon^{n m j} \partial_{j} A_{m}=\frac{1}{2} \varepsilon^{n m j} \partial_{j} \bar{h}_{0 m}
\end{aligned}
$$

one obtains

$$
\left\{\begin{array}{l}
\partial_{i} E^{i}=-4 \pi G \rho \\
\partial_{0} E_{i}-\frac{1}{2} \varepsilon_{i n k} \partial^{k} B^{n}=\frac{4 \pi G}{c} j_{i}
\end{array}\right.
$$

The four gravitomagnetic equations in CGS unit are

$$
\begin{aligned}
\nabla \cdot \mathbf{E} & =-4 \pi G \rho_{m} \\
\nabla \times \mathbf{B} & =-\frac{4 \pi G}{c} \mathbf{J}_{\mathbf{m}}+\frac{\partial \mathbf{E}}{\partial t} \\
\nabla \cdot \mathbf{B} & =0 \\
\nabla \times \mathbf{E}+\frac{\partial \mathbf{B}}{\partial t} & =0
\end{aligned}
$$

where, $\rho_{m}$ is the mass-energy density and $J_{m}$ is the mass current given by: $J_{m}=$ $\rho_{m} v$.

\subsection{Lorentz Force}

The geodesic equations in the weak field approximation is

$$
\frac{d^{2} x^{\mu}}{d s^{2}}+\frac{1}{2} \eta^{\mu \sigma}\left(\partial_{\rho} h_{\nu \sigma}+\partial_{\nu} h_{\rho \sigma}-\partial_{\sigma} h_{\rho \nu}\right) \frac{d x^{\rho}}{d s} \frac{d x^{\nu}}{d s}=0
$$

from where one obtains:

$$
\frac{d^{2} x^{0}}{d s^{2}}+\frac{1}{2} \eta^{00}\left(\partial_{\rho} h_{\nu 0}+\partial_{\nu} h_{\rho 0}-\partial_{0} h_{\rho \nu}\right) \frac{d x^{\rho}}{d s} \frac{d x^{\nu}}{d s}=0,
$$

making the nonrelativisitic limit and an approximation for static fields, which is considered the static gravitational field, we get

$$
0=\frac{d^{2} x^{i}}{d\left(x^{0}\right)^{2}}+\eta^{i j}\left(-\frac{1}{4} \partial_{j} \bar{h}_{00}\right)+\eta^{i j}\left(\partial_{k} \bar{h}_{0 j}-\partial_{j} \bar{h}_{0 k}\right) \frac{d x^{k}}{d x^{0}}
$$

which leads to the gravitomagnetic Lorentz force (15)

$$
F=m\left(\mathbf{E}+\frac{\mathbf{v}}{c} \times 4 \mathbf{B}\right)
$$

\section{Gravitomagnetic Approach in the Solar System}

The GM is expected according to Einstein's General Theory of Relativity, when a planet, star or something massive rotates on its own axis, drag space-time to its turn: this action is called "frame dragging". Some researchers ${ }^{5,6,3}$ affirm that the 
GM can precess satellites in orbits, and this could make a gyroscope (for example, what is in Earth's orbit) balance. With GM concepts and formalism in mind, in this section we calculate the interaction produced by two dipoles to measure the influence of the Sun on Mercury, which is the closest planet to the Sun of the Solar System. Taking into account the gravitomagnetic field that the Sun and Mercury produce as they revolve around themselves we infer the influences of the dipoledipole interaction in the advance of the perihelion of Mercury.

\subsection{Gravitomagnetic Dipole Moment}

According ${ }^{7}$ the vector potential due to a gravitomagnetic dipole $\mu_{g}$ is

$$
\mathbf{A}=-G \frac{\mu_{\mathrm{g}} \times \hat{r}}{|\mathbf{r}|^{3}}
$$

The dipole moment for a uniform distribution of mass in a sphere with constant angular velocity, is given by

$$
\mu_{g}=\frac{M \omega R^{2}}{5 c} \hat{e}_{z}
$$

where, $M$ is sphere mass, $R$ the radius and $\omega$ the angular velocity. In this way, the Gravitomagnetic dipole of a sphere is directly related to its spin by

$$
\mu_{g}=\frac{1}{2 c} \mathbf{S}
$$

where $\mathbf{S}$ is the spin of a rigid and uniform sphere rotating about its axis with angular velocity $\omega$.

\subsection{Gravitomagnetic Field}

The magnetic field due to the dipole $\mu_{g}$ can be calculated from the rotational of the potential vector

$$
\nabla \times \mathbf{A}=-\nabla G \times\left[\frac{\left(\mu_{g} \times \mathbf{r}\right)}{r^{3}}\right]
$$

Then, the gravitomagnetic field components at a distance $r>R$, being $R$ the radius of the sphere, are

$$
\begin{aligned}
\mathbf{B}_{\odot, r} & =-G \frac{2 \mu_{\odot} \cos \theta}{r^{3}} \hat{e}_{r} \\
\mathbf{B}_{\odot, \theta} & =-G \frac{\mu_{\odot} \sin \theta}{r^{3}} \hat{e}_{\theta} \\
\mathbf{B}_{\odot, \phi} & =0 \hat{e}_{\phi}
\end{aligned}
$$

where, $\hat{e}_{r}, \hat{e}_{\theta}$ and $\hat{e}_{\phi}$ are unit vectors, $\mu_{\odot}$ is the module of the gravitomagnetic dipole moment of the Sun and $r$ is the distance between Sun and the planets. 


\section{Potential and Force of Interaction Between Dipoles}

Now we will calculate the expression for the force of interaction between dipoles. For this, we use the expression for the potential energy of a dipole $\mu$ in the gravitomagnetic field of a dipole

$$
U=-4\left(\mu \cdot \mathbf{B}_{\odot}\right)
$$

then the potential becomes

$$
U=-G\left[-\frac{4 \mu_{p} \mu_{\odot}}{r^{3}}\left(2 \cos ^{2} \theta-\sin ^{2} \theta\right)\right]
$$

and the force in the plane of the orbit, i.e. $\theta=\pi / 2$, is

$$
\mathbf{F}_{\mathbf{d}}=-G \frac{12 \mu_{p} \mu_{\odot}}{r^{4}} \hat{e}_{r}
$$

Therefore, the force has a dependence on the radial component and in addition it is an attractive central force. In the following table we show the dipole moment obtained by (21), the gravitomagnetic field and gravitomagnetic forces. We also present have the ratio between the dipole force and the gravitational for the Solar System planets to show how small it is.

\begin{tabular}{l|l|c|l|l} 
Planet & $\mu_{\mathbf{g}}(\mathrm{gcm})$ & $\mathbf{B}\left(\mathrm{cms}^{-2}\right)$ & $\mathbf{F}_{\mathbf{d}}($ dynes $)$ & $F_{d} / F_{\text {grav }}$ \\
\hline Sun & $1.70 \times 10^{38}$ & - & - & - \\
Mercury & $1.08 \times 10^{26}$ & $5.90 \times 10^{-8}$ & $1.32 \times 10^{7}$ & $1.02 \times 10^{-20}$ \\
Venus & $3.56 \times 10^{27}$ & $9.10 \times 10^{-9}$ & $3.60 \times 10^{7}$ & $6.52 \times 10^{-21}$ \\
Earth & $1.18 \times 10^{30}$ & $3.42 \times 10^{-9}$ & $3.24 \times 10^{9}$ & $9.18 \times 10^{-19}$ \\
Mars & $3.50 \times 10^{28}$ & $9.68 \times 10^{-10}$ & $1.78 \times 10^{7}$ & $1.10 \times 10^{-19}$ \\
Jupiter & $1.14 \times 10^{35}$ & $2.43 \times 10^{-11}$ & $4.26 \times 10^{11}$ & $1.03 \times 10^{-17}$ \\
Saturn & $2.35 \times 10^{34}$ & $1.30 \times 10^{-12}$ & $7.74 \times 10^{9}$ & $2.10 \times 10^{-18}$ \\
Uranus & $3.70 \times 10^{32}$ & $4.84 \times 10^{-13}$ & $7.47 \times 10^{6}$ & $5.37 \times 10^{-20}$ \\
Neptune & $4.53 \times 10^{32}$ & $1.25 \times 10^{-13}$ & $1.51 \times 10^{6}$ & $2.27 \times 10^{-20}$
\end{tabular}

\section{Precession of Mercury Using Gravitomagnetic Correction}

\subsection{Gravitomagnetic Dipole-Dipole Potential}

Now we will calculate the advance of the perihelion by adding the gravitomagnetic correction, in which it is assume that the Sun and the planet revolve around their axes. In this way, we will have an effective potential energy given by:

$$
V(r)=-\frac{G M}{r}+\frac{l^{2}}{2 r^{2}}-\frac{k}{r^{3}}
$$

where, $r$ is the distance Sun-planet. $K$ is constant given by $k=\frac{4 G \mu_{p} \mu \odot}{c}$ in accordance with (28). The system energy is:

$$
E=\frac{1}{2}\left(\frac{d r}{d t}\right)^{2}-\frac{G M}{r}+\frac{l^{2}}{2 r^{2}}-\frac{k}{r^{3}}
$$


And now resolving perturbatively as done $i^{8,9}$, we found the advance using the dipole potential, that is

$$
\Delta \phi=2 \pi \frac{6 k}{r_{c} l^{2}}
$$

To Mercury, which is the closest planet to the Sun, the $\Delta \phi$ is approximately $1.49 \times$ $10^{-14} \operatorname{arcsec} / \mathrm{Cy}$.

\section{Conclusion}

We conclude, as expected from the Kepler Laws, that gravitomagnetic effects in the the Solar System is despicable and that the orbits of the planets around the Sun are well described only by the "electric" force of gravitomagnetism i.e. gravitational force of Newton. As a consequence of this result the advancement of Mercury's orbit around the Sun cannot be explained by gravitomagnetism, hence, it is a non-linear effect of the Einstein equations of gravitation.

\section{References}

1. T. Moore, A General Relativity Workbook (Blurb, Incorporated, 2015).

2. R. D'Inverno, Introducing Einsteins Relativity (Clarendon Press, 1992).

3. A. Karlsson, 2006. A gravitomagnetic thought experiment for undergraduates. Technical Report LUTEDX/(TEAT-7150)/1-7/(2006).

4. J. Lense and H. Thirring, Phys. Z. 19, p. 156 (English translation by B. Mashhoon, F. W. Hehl, D. S. Theisis, Gen. Rel. Grav. 16, 711 (1984)).

5. I. Ciufolini and E. C. Pavlis, Nature 431, 958 (2004).

6. L. Iorio, H. I. Lichtenegger, M. L. Ruggiero, and C. Corda, Astrophysics and Space Science 331, 351 (2011).

7. J. D. Jackson, Classical electrodynamics, 3rd ed. edn. (Wiley, New York, NY, 1999).

8. F. Rocha, M. Malheiro, and R. M. Marinho Jr, AIP Conference Proceedings 1693 (2015).

9. F. Rocha, M. Malheiro, and R. M. Marinho Jr, Journal of Physics: Conference Series 706, p. 052014 (2016). 